\title{
Sulfonylurea Receptor 1 in the Germinal Matrix of Premature Infants
}

\author{
J. MARC SIMARD, RUDOLPH J. CASTELLANI, SVETLANA IVANOVA, MICHAEL T. KOLTZ, \\ AND VOLODYMYR GERZANICH
}

\author{
Department of Neurosurgery [J.M.S., S.I., M.T.K., V.G.], Department of Pathology [J.M.S., R.J.C.], Department of Physiology [J.M.S.], \\ University of Maryland School of Medicine, Baltimore, Maryland 21201
}

\begin{abstract}
Germinal matrix (GM) hemorrhage (GMH) is a major cause of mortality and of life-long morbidity from cerebral palsy. GMH is typically preceded by hypoxic/ischemic events and is believed to arise from rupture of weakened veins in the GM. In the CNS, hypoxia/ischemia up-regulate sulfonylurea receptor 1 (SUR1)regulated $\mathrm{NC}_{\mathrm{Ca}-\mathrm{ATP}}$ channels in microvascular endothelium, with channel activation by depletion of ATP being responsible for progressive secondary hemorrhage. We hypothesized that this channel might be up-regulated in the GM of preterm infants at risk for GMH. Here, we studied expression of the regulatory subunit of the channel, SUR1, and its transcriptional antecedent, hypoxia inducible factor 1 (HIF1), in postmortem tissues of premature infants who either were at risk for or who sustained GMH. We found regionally specific up-regulation of HIF1 and of SUR1 protein and mRNA in GM tissues, compared with remote cortical tissues. Up-regulation was prominent in most progenitor cells, whereas in veins, SUR1 was found predominantly in infants who had sustained GMH compared with those without hemorrhage. Our data suggest that the SUR1regulated $\mathrm{NC}_{\mathrm{Ca}-\mathrm{ATP}}$ channel may be associated with $\mathrm{GMH}$, and that pharmacological block of these channels could potentially reduce the incidence of this devastating complication of prematurity. (Pediatr Res 64: 648-652, 2008)
\end{abstract}

$\mathrm{T}_{\mathrm{v}}^{\mathrm{k}}$ he neuropathology underlying cerebral palsy includes white matter injury, such as periventricular leukomalacia and germinal matrix $(\mathrm{GM})$ hemorrhage $(\mathrm{GMH})(1,2)$. Each has distinctive features, but both share important risk factors, including prematurity and hypoxia/ischemia, which may occur prenatally or may be due to postnatal ventilatory difficulties that are complicated by mild-to-moderate hypotension $(1,3,4)$.

GMH is a common complication of prematurity, occurring in $15-45 \%$ of premature infants (3). GMH may range in severity from subependymal hemorrhage (grade 1) to intraventricular hemorrhage without (grade 2) or with (grade 3) ventricular dilatation, to periventricular venous infarction (grade 4). In survivors, neurologic sequelae, particularly with higher grade GMH, include cerebral palsy, hydrocephalus requiring ventricular shunting, learning disabilities, and sei-

Received February 1, 2008; accepted July 9, 2008.

Correspondence: J. Marc Simard, M.D., Ph.D., Department of Neurosurgery, 22 S. Greene Street, Suite 12SD, Baltimore, MD 21201-1595; e-mail: msimard@smail.umaryland.edu

Supported by Grants from the National Heart, Lung and Blood Institute (HL082517), the National Institute of Neurological Disorders and Stroke (NS048260), the Department of Veterans Affairs (Baltimore, MD), and the Christopher and Dana Reeve Foundation (J.M.S.).

J.M.S. holds a US patent (\#7,285,574), "A novel nonselective cation channel in neural cells and methods for treating brain swelling." zures $(5,6)$. Numerous factors are believed to contribute to $\mathrm{GMH}$, including innate weakness of GM veins, autoregulatory dysfunction, hypoxic/ischemic tissue damage, damage due to postischemic reperfusion and increased venous pressure $(4,7-$ 11). The incidence of GMH increases with the degree of prematurity (3), suggesting that advances in perinatal care that yield concomitant increases in the number of extremely premature infants will continue to be hampered by complications of GMH. At present, no effective prevention is available.

Hypoxia/ischemia in rodent and human CNS, both in utero (12) and in adults $(13,14)$, results in up-regulation of sulfonylurea receptor 1 (SUR1). Under pathologic conditions, SUR1 up-regulation is associated with formation of SUR1regulated $\mathrm{NC}_{\mathrm{Ca}-\mathrm{ATP}}$ channels, not $\mathrm{K}_{\mathrm{ATP}}$ channels (13-15). Expression of SUR1-regulated $\mathrm{NC}_{\mathrm{Ca}-\mathrm{ATP}}$ channels in capillary endothelium has been causally implicated in progressive secondary hemorrhage in CNS, with block of these channels by infusion of low-dose (nonhypoglycemogenic) glibenclamide (glyburide) completely preventing secondary hemorrhage (15). Here, we postulated that this channel might be induced in the GM by hypoxia/ischemia, and thereby predispose to GMH. As an initial attempt to assess this hypothesis, we studied expression of the regulatory subunit of the channel, SUR1, and its transcriptional antecedent, hypoxia inducible factor 1 (HIF1) (Bhatta S, Simard JM, unpublished data), in postmortem tissues of premature infants who either were at risk for or who sustained GMH. We report findings consistent with the hypothesis that the SUR1-regulated $\mathrm{NC}_{\mathrm{Ca}-\mathrm{ATP}}$ channel may be causally linked to GMH.

\section{METHODS}

Specimens from premature infants without and with clinically diagnosed GMH were obtained from the Brain and Tissue Bank for Developmental Disorders, University of Maryland, Baltimore, with the collection protocol, including informed consent, reviewed and approved by the Institutional Review Board of the University of Maryland at Baltimore. The postmortem interval was 3-24 h. Cases were selected for study based either on: (i) the documented presence of GMH/intraventricular hemorrhage at autopsy or (ii) documented absence of GMH (used as "best-available" controls). Independent histologic validation of presence or absence of GMH was made in all cases (Table 1). In all but one case, the cause of prematurity was preterm

\footnotetext{
Abbreviations: DAPI, 4',6-diamino-2-phenylindole; GM, germinal matrix; GMH, germinal matrix hemorrhage; H\&E, hematoxylin and eosin; HIF1, hypoxia inducible factor 1; SUR1, sulfonylurea receptor 1
} 
Table 1. Summary of cases examined

\begin{tabular}{|c|c|c|c|c|c|c|}
\hline Case \# & $\begin{array}{l}\text { Gestational age at } \\
\text { birth (weeks) }\end{array}$ & Hemorrhage clinically & $\begin{array}{c}\text { Hemorrhage* in H\&E } \\
\text { section }\end{array}$ & $\begin{array}{l}\text { HIF1 protein in } \\
\text { cells } \dagger\end{array}$ & $\begin{array}{l}\text { SUR1 protein in } \\
\text { cellsł }\end{array}$ & $\begin{array}{l}\text { SUR1 protein in } \\
\text { veins } \S\end{array}$ \\
\hline 1 & 19 & None & None & + & +++ & 0 \\
\hline 2 & 19 & None & None & ++ & + & $0 /+$ \\
\hline 3 & 22 & None & None & ++ & +++ & + \\
\hline 4 & 22 & None & None & +++ & ++ & + \\
\hline 5 & 22 & None & None & +++ & + & $+/++$ \\
\hline 6 & 23 & None & Microscopic & ++ & + & $+/++$ \\
\hline 7 & 24 & None & Microscopic & +++ & +++ & $+1++$ \\
\hline 8 & 24 & Grade 1 & Grade 1 & +++ & +++ & ++++ \\
\hline 9 & 22 & Grade 1 & Grade 1 & ++++ & ++++ & ++++ \\
\hline 10 & 24 & Grade $2 / 3$ & None & ++++ & ++++ & ++++ \\
\hline 11 & 30 & Grade $2 / 3$ & Grade 1 & +++++ & +++ & ++++ \\
\hline 12 & 23 & Grade $2 / 3$ & Grade $2 / 3$ & +++++ & ++ & +++ \\
\hline
\end{tabular}

* Clinical information was available only on "intraventricular hemorrhage" without differentiating further into grade, hence the designation, grade $2 / 3$; some discrepancies in clinical vs. histological evaluation of hemorrhage may be due to histological evaluation of the GM contralateral to the side of hemorrhage, which available data were insufficient to resolve.

$\dagger$ Scale for HIF1 immunolabeling in progenitor cells within the GM: + , present in most cells, similar in intensity to some distant neurons; ++ , present in most cells, somewhat more intense than in neurons; +++ , present in most cells, definitely more intense than in neurons; ++++ , present in all cells, more intense than in neurons; +++++ , present in all cells, many with very intense labeling.

\$ Scale for SUR1 immunolabeling in progenitor cells within the GM: + , present in few single cells; ++ , present in a moderate number of scattered cells; +++ , present in patches or groups of cells; ++++ present in most cells.

$\S$ Scale for SUR1 immunolabeling in veins within the GM: 0 , none; + , in $1-2$ veins; ++ , in a few veins; +++ , in many veins; ++++ , in nearly all veins.

rupture of membranes, with some cases also documenting chorioamnionitis by pathologic examination of the placenta, and one case (without GMH) being induced for cardiac anomaly. The cause of death was extreme prematurity in all but two cases, with the others being listed as amniotic fluid aspiration syndrome or elective termination.

GM tissues and associated hemorrhages, when present, were dissected from coronal slices of formalin-fixed cerebral hemispheres. Cryosections and paraffin-embedded sections were prepared. Sections were stained with hematoxylin and eosin $(\mathrm{H} \& \mathrm{E})$ or were immunolabeled using primary antibodies directed against SUR1 (C-16; Santa Cruz Biotechnology Inc.; diluted 1:200; $1 \mathrm{~h}$ at room temperature, $48 \mathrm{~h}$ at $4^{\circ} \mathrm{C}$ ), or HIF- $1 \alpha$ (SC-10790; Santa Cruz; 1:100), or von Willebrand factor (F-3520; Sigma Chemical Co.; 1:200). CY-3 or FITC conjugated secondary antibodies (Jackson Immunoresearch, West Grove, PA) were used. Slides were cover slipped with ProLong Gold antifade reagent containing 4',6-diamino-2-phenylindole (DAPI) for nuclear staining (P36935, Invitrogen, Carlsbad, CA). For in situ hybridization, digoxigeninlabeled probes (antisense, 5' -TGCAGGGGTCAGGGTCAGGGcGCTGTCGGTCCACTTGGCCAGCCAGTA-3'), designed to hybridize to nucleotides 3217-3264 located within coding sequence of human Abcc8 gene (NM 00352), were supplied by GeneDetect (Brandenton, FL). Hybridization was performed according to the manufacturer's protocol, as previously described (13).

\section{RESULTS}

The GM appeared as a dense collection of small cells located peri-ventricularly (Fig. $1 A$ ). In some cases, evidence of a parenchymal hemorrhage was found (Fig. 1A, arrow).

In situ hybridization for mRNA for $A b c c 8$, which encodes SUR1, showed regionally specific up-regulation in the GM (Fig. $1 B$ and $E$ ) that was noticeably more prominent than in surrounding tissues or in remote cortical tissues (Fig. 1D). Immunolabeling confirmed regionally specific up-regulation of SUR1 protein in the GM (Fig. 1C), with SUR1 protein located in neural progenitor cells in all GM specimens examined (Fig. $1 G$ ). SUR1 protein was also identified in veins from infants with GMH (Fig. $1 H$ and $I$ ), but was less likely to be found in veins from infants without GMH (Fig. 1J). Negative controls, including omission of primary antibody and use of a blocking peptide, showed no immunolabeling for SUR1 (not shown).
An important molecular antecedent of SUR1 is the transcription factor, HIF1 (Bhatta S, Simard JM, unpublished data), which is up-regulated by hypoxia (16), a common condition associated with prematurity. Immunolabeling for HIF1 $\alpha$ showed that this ubiquitous marker of hypoxia was prominently up-regulated, with characteristic nuclear localization, in all GM specimens examined (Fig. $1 K-M$ ).

We performed a semiquantitative assessment of HIF $1 \alpha$ and SUR1 expression in specimens from 12 premature infants, some of whom had either clinical or histologic evidence of GMH (Table 1). All specimens showed HIF1 $\alpha$ expression, with all but one showing more prominent expression in progenitor cells than in remote neurons in the same tissue sections, supporting the hypothesis that physiologically meaningful hypoxia was present in the GM of all of these cases. The most prominent expression of HIF1 $\alpha$ was found in specimens from infants with frank GMH. All specimens showed SUR1 expression in progenitor cells. In three specimens, SUR1 was identified only in scattered cells, whereas in most specimens, SUR1 expression was evident in contiguous sheets of cells or in some cases, in nearly all cells. The clearest distinction in SUR1 expression vis-à-vis GMH was in the veins of the GM. In specimens without GMH, the veins typically exhibited little to moderate SUR1 expression, as in Fig. $1 J$, whereas in all specimens with frank GMH, all or nearly all veins exhibited strong SUR1 expression, as in Fig. $1 H$ and $I$.

\section{DISCUSSION}

We provide evidence that expression of SUR1 is increased in neural progenitor cells and in vascular endothelium of the GM of premature infants who either are at risk for or who sustained GMH. Immunohistochemical analysis of postmortem tissues can sometimes be complicated by nonspecific binding of antibodies, especially if necrosis is present. How- 


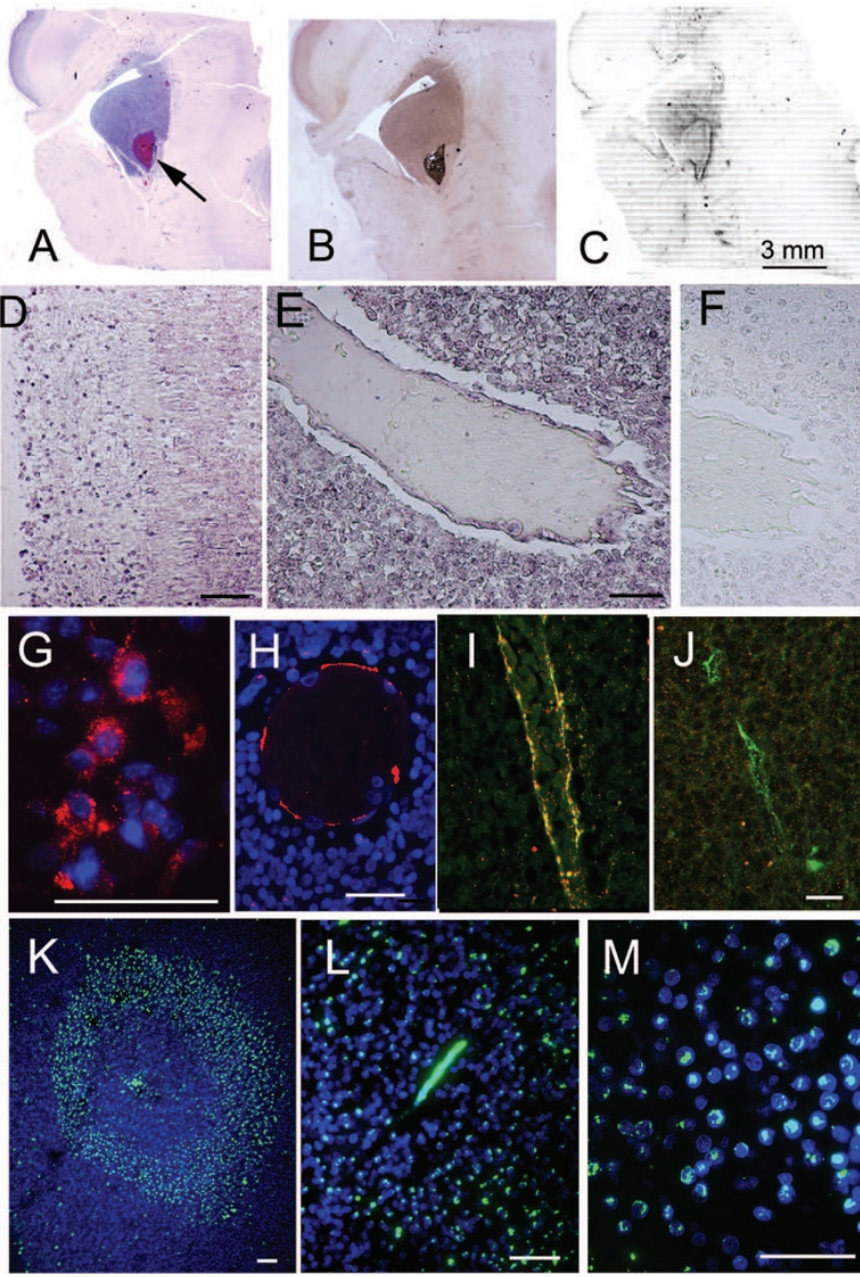

Figure 1. SUR1 and HIF1 are up-regulated in the germinal matrix of premature infants. $A-C$, Low power micrographs $(A, B)$ or montage of micrographs $(C)$ of periventricular tissue stained with $\mathrm{H} \& \mathrm{E}(A)$, showing densely packed neural progenitor cells of the GM, with an arrow pointing to a small intraparenchymal hematoma, or labeled for mRNA for $A b c c 8$, which encodes SUR1, using in situ hybridization $(B)$, or immunolabeled for SUR1 (C); the latter two demonstrate regionally-specific labeling for SUR1 mRNA and protein in the GM; the montage in $(C)$ shows positive immunolabeling in black pseudocolor; case \#9 in Table 1: premature infant of $22 \mathrm{wk}$ gestation who lived $\sim 12 \mathrm{~h}$ and was hypoxic before death, necessitating intubation and mechanical ventilation; postmortem interval, 3 h. $D-F$, Micrographs of cortical tissues $(D)$ or GM tissues $(E, F)$ processed for in situ hybridization for mRNA for $A b c c 8$, using antisense probe $(D, E)$ or sense probe $(F) . G-J$, Micrographs of GM tissues immunolabeled for SUR1 (red, CY3 for SUR1, and blue, DAPI for nuclei), and double-labeled for von Willebrand factor (green; panels I and J only); co-labeling is indicated by yellow color; SUR1 was identified in neural progenitor cells $(G)$, and in thin-walled veins from infants with GMH (panel $H$, red and panel I, yellow) but not in an infant without GMH (panel $J$, green); panels $H-J$ are from cases \#11, 10, 1 in Table 1 , respectively. $K-M$, Low $(K)$ and high $(L, M)$ power micrographs of sections immunolabeled for HIF1 $\alpha$ (green, FITC for HIF1 $\alpha$, and blue, DAPI for nuclei), showing HIF1 $\alpha$ in a microvessel $(L)$ and in neural progenitor cells $(M)$. In panels $D-M$, the bars represent $50 \mu \mathrm{m}$.

ever, the specimens we studied showed intact cellular structures with H\&E staining, as well as regionally-specific immunolabeling of cellular and vascular structures for SUR1 in the GM. Most importantly, we used in situ hybridization to confirm that SUR1 was up-regulated at the mRNA level. Together, the two independent techniques using molecularly distinct probes provide important corroborative evidence that SUR1 was up-regulated in GM tissues of premature infants. Additional work will be required to demonstrate concomitant up-regulation of the pore-forming subunit of the channel (14).

Pathophysiology. The pathophysiological antecedents of GMH have been extensively discussed, but no fully encompassing theory has been put forth to explain it. Considerable attention has been focused on the structural weakness of GM microvessels $(8,9)$. However, it is evident that any innate weakness of these vessels, by itself, would be insufficient to cause GMH, since the same weakness exists during every gestation, and most gestations are not complicated by GMH. Thus, an event must transpire to weaken these vessels further and increase the likelihood of their structural failure. In the premature brain, the GM is at the terminal end of its afferent arteriolar supply ("ventriculopetal" vascular pattern) (17) and, therefore, GM tissues and the vessels contained therein are highly susceptible to global hypoxic/ischemic events. Apart from hypoxia due to ventilatory abnormalities, one or more hypotensive episodes may contribute to the overall hypoxic/ischemic burden that adversely affects GM tissues. In addition, it is likely that yet another hemodynamic stress must be applied to structurally compromised vessels to cause an actual GMH. Because GMH most frequently arises from veins $(7,11)$, it is thought that episodes of increased venous pressure, as can occur with mechanical ventilation or airway suctioning, may be important for triggering the actual structural failure of weakened vessels that results in GMH. Despite the important role of hypoxia/ischemia in producing vascular changes that predispose to $\mathrm{GMH}$, there is little experimental evidence to elucidate the molecular mechanism involved, either in animal models or in humans. To our knowledge, ours is the first report to show that the transcription factor, HIF1, is up-regulated in the GM of infants at risk. In many organs including the CNS, hypoxia results in activation of HIF1, which in turn stimulates the transcription of genes that are essential for adaptation to hypoxia/ischemia, including genes important for erythropoiesis, glycolysis and angiogenesis (16). HIF1 plays a critical role in expression of the angiogenic factor, vascular endothelial growth factor, which is prominently up-regulated in the GM of infants at risk (18). Conversely, HIF1 also causes transcription of genes with seemingly maladaptive effects (19) and, in some settings, may promote ischemia-induced neuronal death (20). HIF1 has not been extensively studied in the premature infant brain, and a role for HIF1 has not previously been suggested in the context of GMH. However, the localization of HIF1 with two of its important transcriptional targets, vascular endothelial growth factor (18) and SUR1, in the GM of infants at risk reaffirms the importance of this molecular response to hypoxia.

Events in the GM. Mild hypoxia activates quiescent neural progenitor cells, resulting in their activation and differentiation into neurons and glia, whereas severe hypoxia induces apoptotic death in developing brain neurons 


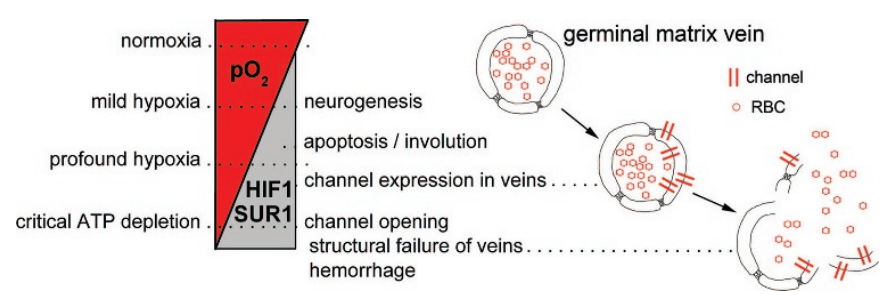

Figure 2. Events in the germinal matrix of premature infants. Scheme depicting the reciprocal relationship between $\mathrm{O}_{2}$ tension on the one hand, and HIF1 activation and SUR1 expression on the other hand. Mild hypoxia, which may be the norm due to the ventriculopetal blood supply, promotes neurogenesis, whereas moderate hypoxia may promote apoptosis resulting in involution of the GM. More severe hypoxia may promote expression of SUR1-regulated $\mathrm{NC}_{\mathrm{Ca}-\mathrm{ATP}}$ channels, which remain inactive until critical ATP depletion is reached $(\sim 30 \mu \mathrm{M})$, at which point the channels open, leading to oncotic death of cells, including endothelial cells, thereby compromising the structural integrity of veins and predisposing to GMH during episodes of venous hypertension.

(21). Thus, mild-to-moderate hypoxia, resulting from the position of the GM as the distant-most tissue fed by a ventriculopetal blood supply (17), may be involved not only in stimulating neurogenesis from GM progenitor cells, but also in the normal involution of the GM (Fig. 2). HIF1, the ubiquitous sensor of hypoxia, may be a key molecular participant in both. Notably, the same hypoxic signal working via HIF1 also leads to transcriptional up-regulation of SUR1 (Bhatta S, Simard JM, unpublished data) and of SUR1-regulated $\mathrm{NC}_{\mathrm{Ca}-\mathrm{ATP}}$ channels (15). In all of the 12 cases studied, most of the progenitor cells exhibited both HIF1 and SUR1, suggesting that mild hypoxia may be a normal state in GM parenchyma, and that this tissue may be normally "primed" with SUR1. When the $\mathrm{NC}_{\mathrm{Ca}-\mathrm{ATP}}$ channel is expressed in response to an hypoxic stimulus, no adverse functional consequence is expected, as long as intracellular ATP is maintained at sufficient levels $(>30$ $\mu \mathrm{M})$ to keep the channel from opening (14). Under conditions of extreme duress, a normal hypoxic signal may be magnified by one or more ischemic events, leading to more profound hypoxia. Under such conditions, HIF1 activation and SUR1 expression would become more likely, especially in veins (Fig. 2). Normally, cells of the vascular tree are less likely than parenchymal cells to experience hypoxia, but under conditions of extreme duress, when maximum extraction of $\mathrm{O}_{2}$ has already occurred from hypoxic blood, venular cells will experience the strongest hypoxic challenge. In the cases we studied, veins generally were less likely to exhibit SUR1 than parenchymal cells, but in cases with GMH, SUR1 expression was reliably found in most veins - the very structures that are believed to be the source of hemorrhage $(7,11)$. When ATP is depleted to critical levels, SUR1-regulated $\mathrm{NC}_{\mathrm{Ca}-\mathrm{ATP}}$ channels open, leading to oncotic cell death (13) not only of progenitor cells but also of vascular endothelial cells, thereby further weakening thin walled, structurally compromised veins. In this setting, increased venous pressure would almost certainly cause extravasation of blood from damaged veins. Petechial hemorrhages may enlarge to microhemorrhages or grade $1 \mathrm{GMH}$, or worse, depending on the severity and extent of GM tissues involved. We believe that this sequence (Fig. 2), postulating critical involvement of HIF1 and SUR1, accounts for numerous observations and encompasses numerous hypotheses that have been put forth to explain GMH.

Preventing GMH. Available strategies for preventing GMH are limited. Currently, the most effective measures are those that target the respiratory system $(22,23)$. Vitamin E, phenobarbital, morphine, ibuprofen, indomethacin, agents that target coagulation, and magnesium/aminophylline have been tried, but are either ineffective or their use remains controversial. In an animal model of GMH, prenatal treatment with angiogenic inhibitors reduces the incidence of GMH (18), but angiogenic suppression in premature infants would be undesirable, since it could impair lung maturation (24). Novel treatment strategies are desperately needed to combat GMH. Block of SUR1 using glibenclamide may be such a treatment. Glibenclamide pretreatment in humans is associated with significantly better outcomes from stroke $(14,25)$, and constant infusion of drug at doses below those that give hypoglycemia is highly effective in preventing progressive secondary hemorrhage in the CNS (15). The findings reported here are consistent with the hypothesis that the SUR1-regulated $\mathrm{NC}_{\mathrm{Ca}-\mathrm{ATP}}$ channel may be causally linked to GMH. If corroborated, involvement of the channel would suggest that glibenclamide might be useful in reducing the incidence of this devastating complication of prematurity.

\section{REFERENCES}

1. Vergani P, Locatelli A, Doria V, Assi F, Paterlini G, Pezzullo JC, Ghidini A 2004 Intraventricular hemorrhage and periventricular leukomalacia in preterm infants. Obstet Gynecol 104:225-231

2. Folkerth RD 2005 Neuropathologic substrate of cerebral palsy. J Child Neurol 20:940-949

3. Kadri H, Mawla AA, Kazah J 2006 The incidence, timing, and predisposing factors of germinal matrix and intraventricular hemorrhage (GMH/IVH) in preterm neonates. Childs Nerv Syst 22:1086-1090

4. Lou HC 1993 On the pathogenesis of germinal layer hemorrhage in the neonate. APMIS Suppl 40:97-102

5. Levy ML, Masri LS, McComb JG 1997 Outcome for preterm infants with germinal matrix hemorrhage and progressive hydrocephalus. Neurosurgery 41:1111-1117

6. Pikus HJ, Levy ML, Gans W, Mendel E, McComb JG 1997 Outcome, cost analysis, and long-term follow-up in preterm infants with massive grade IV germinal matrix hemorrhage and progressive hydrocephalus. Neurosurgery 40:983-988

7. Nakamura Y, Okudera T, Fukuda S, Hashimoto T 1990 Germinal matrix hemorrhage of venous origin in preterm neonates. Hum Pathol 21:1059-1062

8. Wei W, Xin-Ya S, Cai-Dong L, Zhong-Han K, Chun-Peng C 2000 Relationship between extracellular matrix both in choroid plexus and the wall of lateral ventricles and intraventricular hemorrhage in preterm neonates. Clin Anat $13: 422-428$

9. Anstrom JA, Brown WR, Moody DM, Thore CR, Challa VR, Block SM 2004 Subependymal veins in premature neonates: implications for hemorrhage. Pediatr Neurol 30:46-53

10. Berger R, Garnier Y, Jensen A 2002 Perinatal brain damage: underlying mechanisms and neuroprotective strategies. J Soc Gynecol Investig 9:319-328

11. Ghazi-Birry HS, Brown WR, Moody DM, Challa VR, Block SM, Reboussin DM 1997 Human germinal matrix: venous origin of hemorrhage and vascular characteristics. AJNR Am J Neuroradiol 18:219-229

12. Xia Y, Eisenman D, Haddad GG 1993 Sulfonylurea receptor expression in rat brain: effect of chronic hypoxia during development. Pediatr Res 34:634-641

13. Simard JM, Chen M, Tarasov KV, Bhatta S, Ivanova S, Melnitchenko L, Tsymbalyuk N, West GA, Gerzanich V 2006 Newly expressed SUR1-regulated $\mathrm{NC}(\mathrm{Ca}-\mathrm{ATP})$ channel mediates cerebral edema after ischemic stroke. Nat Med 12:433-440

14. Simard JM, Woo SK, Bhatta S, Gerzanich V 2008 Drugs acting on SUR1 to treat CNS ischemia and trauma. Curr Opin Pharmacol 8:42-49

15. Simard JM, Tsymbalyuk O, Ivanov A, Ivanova S, Bhatta S, Geng Z, Woo SK, Gerzanich V 2007 Endothelial sulfonylurea receptor 1-regulated NC Ca-ATP chan- 
nels mediate progressive hemorrhagic necrosis following spinal cord injury. J Clin Invest 117:2105-2113

16. Wenger RH, Stiehl DP, Camenisch G 2005 Integration of oxygen signaling at the consensus HRE. Sci STKE 2005:re12

17. Nakamura Y, Okudera T, Hashimoto T 1994 Vascular architecture in white matter of neonates: its relationship to periventricular leukomalacia. J Neuropathol Exp Neurol 53:582-589

18. Ballabh P, Xu H, Hu F, Braun A, Smith K, Rivera A, Lou N, Ungvari Z, Goldman SA, Csiszar A, Nedergaard M 2007 Angiogenic inhibition reduces germinal matrix hemorrhage. Nat Med 13:477-485

19. Simard JM, Kent TA, Chen M, Tarasov KV, Gerzanich V 2007 Brain oedema in focal ischaemia: molecular pathophysiology and theoretical implications. Lancet Neurol 6:258-268

20. Chang YC, Huang CC 2006 Perinatal brain injury and regulation of transcription. Curr Opin Neurol 19:141-147
21. Pourie G, Blaise S, Trabalon M, Nedelec E, Gueant JL, Daval JL 2006 Mild, non-lesioning transient hypoxia in the newborn rat induces delayed brain neurogenesis associated with improved memory scores. Neuroscience 140:13691379

22. Cools F, Offringa M 2005 Neuromuscular paralysis for newborn infants receiving mechanical ventilation. Cochrane Database Syst Rev: CD002773

23. Wright LL, Horbar JD, Gunkel H, Verter J, Younes N, Andrews EB, Long W 1995 Evidence from multicenter networks on the current use and effectiveness of antenatal corticosteroids in low birth weight infants. Am J Obstet Gynecol 173:263-269

24. Thebaud B 2007 Angiogenesis in lung development, injury and repair: implications for chronic lung disease of prematurity. Neonatology 91:291-297

25. Kunte H, Schmidt S, Eliasziw M, del Zoppo GJ, Simard JM, Masuhr F, Weih M, Dirnagl U 2007 Sulfonylureas improve outcome in patients with type 2 diabetes and acute ischemic stroke. Stroke 38:2526-2530 